\title{
A rapid RP-HPLC method for the simultaneous estimation of Ivacaftor and Tezacaftor and in silico study of their metabolitic products
}

\author{
Madhuri Donakonda ${ }^{1 *}$ D, Srija Indrakanti ${ }^{1}$, Praveen Kumar Pasala ${ }^{2}$, Malleswari Desari ${ }^{1}$ and Shireesha Kammari ${ }^{1}$
}

\begin{abstract}
Background: This study was designed to develop a reliable method for estimation of Ivacaftor and Tezacaftor in pure and its pharmaceutical dosage form by RP-HPLC in human plasma. Molecular docking studies were carried out and the results were visualized using PyMol and Discovery studio visualizer (Discovery studio visualizer ver. 2.5). The pharmacokinetic properties such as Swiss ADME and pKCSM of the Ivacaftor and its metabolites Ivacaftor M1, M6 and Tezacaftor and metabolites Tezacaftor M1, M2 were predicted. In admetSAR, web-based query tools incorporating a molecular built-in interface enable the database to be queried by SMILES.

Results: A simple, linear, precise, and accurate RP-HPLC method was developed and validated for the determination of Ivacaftor (IVA) and Tezacaftor (TEZ) in human plasma. Chromatographic separation was achieved isocratically on Inspire $\mathrm{C} 18,(4.6 \times 250 \mathrm{~mm}, 5 \mu \mathrm{m})$ column at $30^{\circ} \mathrm{C}$. Mobile phase consisting of methanol and $0.05 \%$ formic acid in ratio of 95:5 with flow rate of $1 \mathrm{~mL} / \mathrm{min}$ with injection volume $20 \mu \mathrm{l}$ detector used is PDA at $235 \mathrm{~nm}$. The developed method was validated according to $\mathrm{ICH}$ guidelines and found to be linearity range was found to be for TEZ (10-50 $\mu \mathrm{g} / \mathrm{mL})$ and IVA $(15-75 \mu \mathrm{g} / \mathrm{mL})$. IVA and TEZ drugs and its metabolites were retrieved from the PubChem database and the 2D chemical structures were generated from SMILES notation by using the Chemsketch Software. The structure was viewed using Swiss-PDB Viewer to form a better understanding of the molecule for toxicity and biological activity prediction.
\end{abstract}

Conclusion: The results obtained by the proposed method from validation parameters and from assay confirmed that the determination of Tezacaftor (TEZ) and Ivacaftor (IVA) in their combined dosage form in human plasma was sensitive and selective method. In silico study has revealed that IVA and its metabolites IVA M1, IVA M6 are according to Lipinski rule. The oral bioactivity of IVA was found to be more when compared to its metabolites (Molinspiration) and TEZ and its metabolites TEZ M1, TEZ M2 even though they have the molecular weight > 500, but all other parameters from Molinspiration revealed better oral bioactivity of TEZ M2. Validation of the developed isocratic RP-HPLC procedure revealed that, regardless of how the sample was purified, the method was characterized by good linearity, sensitivity, reproducibility, specificity, and low values of LOD $(0.090 \mu \mathrm{g} / \mathrm{mL})$ and LOQ $(0.275 \mu \mathrm{g} / \mathrm{mL})$. From the in silico docking results, it is quite evident that metabolites of TEZ and IVA have the great potential against cystic fibrosis.

Keywords: Tezacaftor, Ivacaftor, In silico docking, Human plasma, RP-HPLC metabolites

\footnotetext{
* Correspondence: madhurisatyasai@gmail.com

'Department of Pharmaceutical Analysis and Quality Assurance, CES College

of Pharmacy, Chinnatekur, NH-7, Kurnool, Andhra Pradesh 518218, India

Full list of author information is available at the end of the article
}

\section{Springer Open}

( ) The Author(s). 2021 Open Access This article is licensed under a Creative Commons Attribution 4.0 International License, which permits use, sharing, adaptation, distribution and reproduction in any medium or format, as long as you give appropriate credit to the original author(s) and the source, provide a link to the Creative Commons licence, and indicate if changes were made. The images or other third party material in this article are included in the article's Creative Commons licence, unless indicated otherwise in a credit line to the material. If material is not included in the article's Creative Commons licence and your intended use is not permitted by statutory regulation or exceeds the permitted use, you will need to obtain permission directly from the copyright holder. To view a copy of this licence, visit http://creativecommons.org/licenses/by/4.0/. 


\section{Background}

Ivacaftor, (N-(2,4-ditert-butyl-5-hydroxyphenyl)-4oxo-1H-quinoline-3-carboxamide) [1] is used for treatment of cystic fibrosis. It has a role as a CFTR potentiator [2]. Tezacaftor, (1-(2,2-difluoro-1,3-benzodioxol-5-yl)- $N$-[1-[(2R)-2,3-dihydroxypropyl]-6-fluo ro-2-(1-hydroxy-2-methylpropan-2-yl)indol-5-yl] cycl opropane-1-carboxamide) [3] is used as a corrector of CFTR gene function [2]. Symdeko contains combination of Tezacaftor and Ivacaftor. Tezacaftor moves to defective CFTR protein onto the cell surface, while Ivacaftor helps to facilitate the opening of chloride channel on the cell surface to increase chlorine transport [2] (Figs. 1, 2 and 3).

Literature review reveals that analytical methods based on stability indicating studies [4] R.P-HPLC [4-8]. Degradation studies of Ivacaftor [9], stability indicating studies of Ivacaftor and Lumacaftor [10]. HPLC and L.C/M.S [11]. U.V. spectrophotometric method

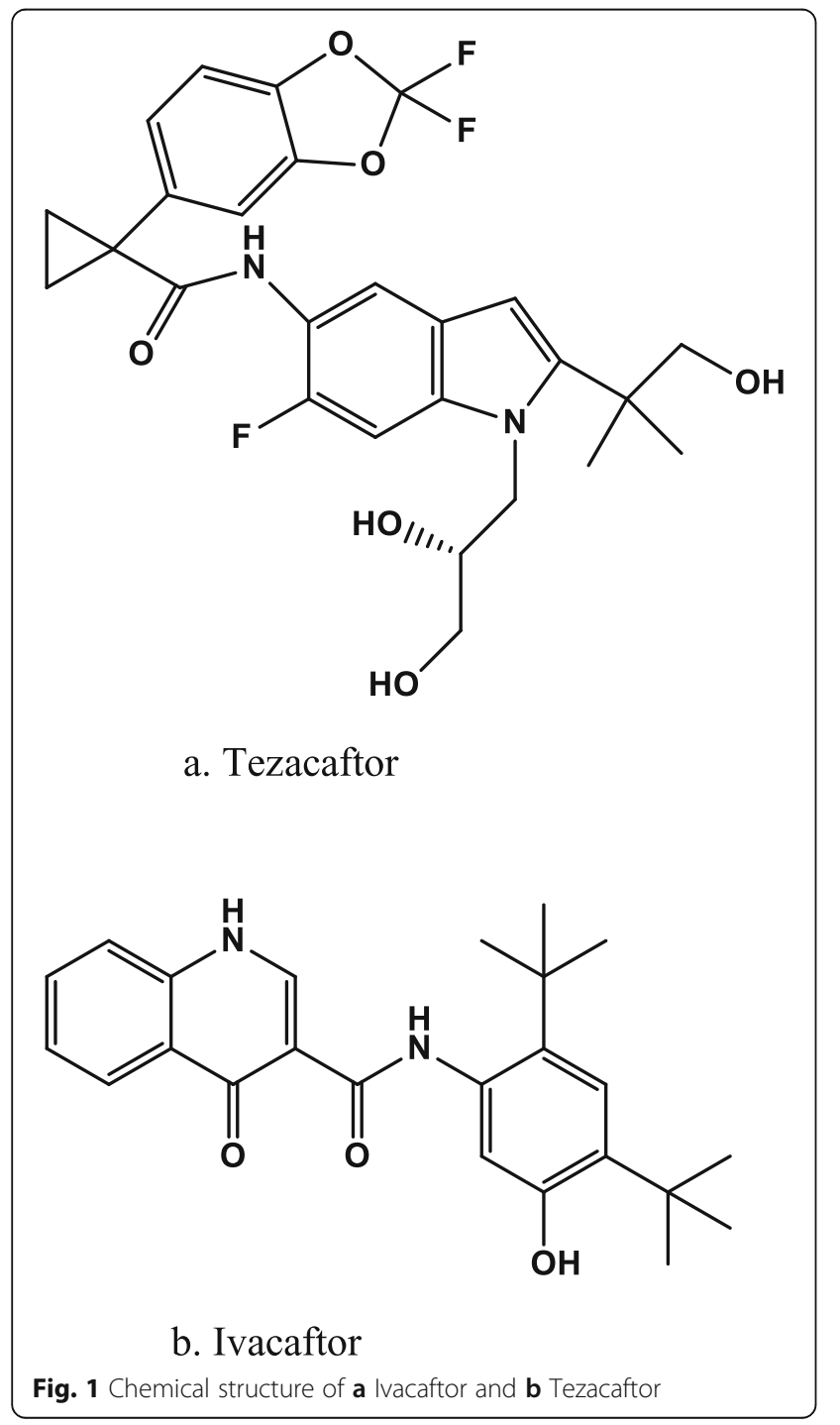

for the determination of Ivacaftor and Tezacaftor [12], UV determination of Ivacaftor [13], stability, and U.P.L.C [14]. Some papers have described the analysis of combination with other drugs in plasma, based on HPLC and L.C/M.S [11]. However, in most of the methods, mobile phase used consists of buffer, and there is no method reported regarding the simultaneous determination of Ivacaftor and Tezacaftor in human plasma.

The fixed dosage combination of two drugs is important for the treatment of pulmonary cystic fibrosis and thus a single precise method capable of separating the two drugs with good resolution using a simple mobile phase in human plasma is time-saving, novel, and rapid. Hence, in present work, we have developed a simple, linear, precise, accurate, and validated HPLC method for determination of Ivacaftor and Tezacaftor in mobile phase and human plasma according to $\mathrm{ICH}$ guidelines. The in silico tools provide a quick study and predict the biological activity as well toxicity associated with its metabolites [15] and give an indication of the pharmacological implications in humans [16-24].

\section{Method}

Pure samples

Ivacaftor and Tezacaftor purity of $99.9 \%$ were received as gift samples from Laurus Labs.

\section{Formulation}

Samples contained mixture of drugs and placebo in appropriate doses of drugs as per the Vortex Pharmaceutical preparation of Symdeko.

\section{Chemicals and reagents}

HPLC grade ACN, methanol, water, and formic acid were purchased from Fischer chemicals Ltd., India. All other reagents employed were of high purity analytical grade. All weighing was done on a calibrated analytical balance. Calibrated glassware was used throughout the work. Fresh human plasma was procured (Blood Group $\mathrm{AB}+\mathrm{VE}$ ) from local blood bank, i.e., Red Cross Society, Bill No. 5703.

\section{RP-HPLC instrumentation and chromatographic conditions}

The HPLC system (Shimadzu, spd-M20 A) equipped with Inspire, C18, $(4.6 \times 250 \mathrm{~mm}, 5 \mu \mathrm{m})$ column component having temperature control, PDA detector, and Rheodyne injector control. U.V. detection was performed at $235 \mathrm{~nm}$ based on UV spectrophotometric scanning from 200 to $400 \mathrm{~nm}$ and was recorded online for peak identification using Lab India (U.V. $3000^{+}$) U.V/Vis spectrophotometer. The 
a) Ivacaftor M1<smiles>CC(C)(C)c1cc(C(C)(C)CO)c(NC(=O)c2c[nH]c3ccccc3c2=O)cc1O</smiles>

(a) b) Ivacaftor M6

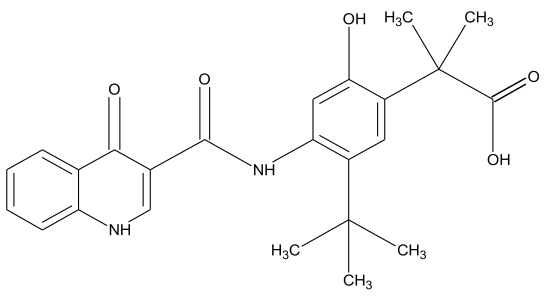

(b)

Fig. 2 Chemical structure of a Ivacaftor M1 and $\mathbf{b}$ Ivacaftor M6

mobile phase consisted of methanol and $0.05 \%$ formic acid in ratio of (95:5). The flow rate is $1.0 \mathrm{~mL} /$ min. The injection volume of sample was $20 \mu \mathrm{L}$. Column temperature was maintained at $30{ }^{\circ} \mathrm{C}$.<smiles>CC1(C)c2cc3cc(NC(=O)C4(c5ccc6c(c5)OC(F)(F)O6)CC4)c(F)cc3n2CC(CO)OC1O</smiles>

a. Tezacaftor M1 Metabolite<smiles>CC(C)(C(=O)O)c1cc2cc(NC(=O)C3(c4ccc5c(c4)OC(F)(F)O5)CC3)c(F)cc2n1C[C@H](O)CO</smiles>

b. Tezacaftor M2 Metabolite

Fig. 3 Chemical structure of a Tezacaftor M1 and $\mathbf{b}$ Tezacaftor M2

\section{Methodology}

Selection of wave length

Accurately weighed $10 \mu \mathrm{g} / \mathrm{mL}$ of Tezacaftor and $15 \mu \mathrm{g} /$ $\mathrm{mL}$ of Ivacaftor were diluted to $10 \mathrm{~mL} \mathrm{ACN}$ solvent. The above solution was scanned between 200 and $400 \mathrm{~nm}$ by UV-Visible spectroscopy (Fig. 4).

\section{Preparation of mobile phase}

Methanol and $0.05 \%$ formic acid in ratio of $95: 5 \mathrm{v} / \mathrm{v}$ proportions were used as mobile phase which were filtered by vacuum filtration using $0.45 \mu$ filter paper and were sonicated for $30 \mathrm{~min}$ prior to use.

\section{Preparation of standard solution}

About $100 \mathrm{mg}$ of Tezacaftor and $100 \mathrm{mg}$ of Ivacaftor were accurately weighed and transferred into $100 \mathrm{~mL}$ volumetric flask separately. ACN was added to ensure complete solubility, and the volume was adjusted with the mobile phase to obtain a concentration of 1000 $\mu \mathrm{g} / \mathrm{mL}$ of TEZ and $1000 \mu \mathrm{g} / \mathrm{mL}$ IVA standard solutions.

\section{Working standard solutions in mobile phase}

Three hundred microliters and $450 \mu \mathrm{L}$ of standard solutions of TEZ and IVA were pipetted out using micropipette into $10 \mathrm{~mL}$ volumetric flask seperately and diluted up to the mark with mobile phase to obtain working standard solutions of final concentrations $30 \mu \mathrm{g} / \mathrm{mL}$ of Tezacaftor and $45 \mu \mathrm{g} / \mathrm{mL}$ of Ivacaftor.

\section{Working standard solution in human plasma (protein precipitation method)}

Ninety microliters of human plasma and $2 \mathrm{~mL}$ of ACN were added from standard solutions, $0.1 \mathrm{~mL}$ of Tezacaftor and $0.15 \mathrm{~mL}$ of Ivacaftor were taken in centrifuge tube. They were vortexed for $1 \mathrm{~min}$ and centrifuged at 


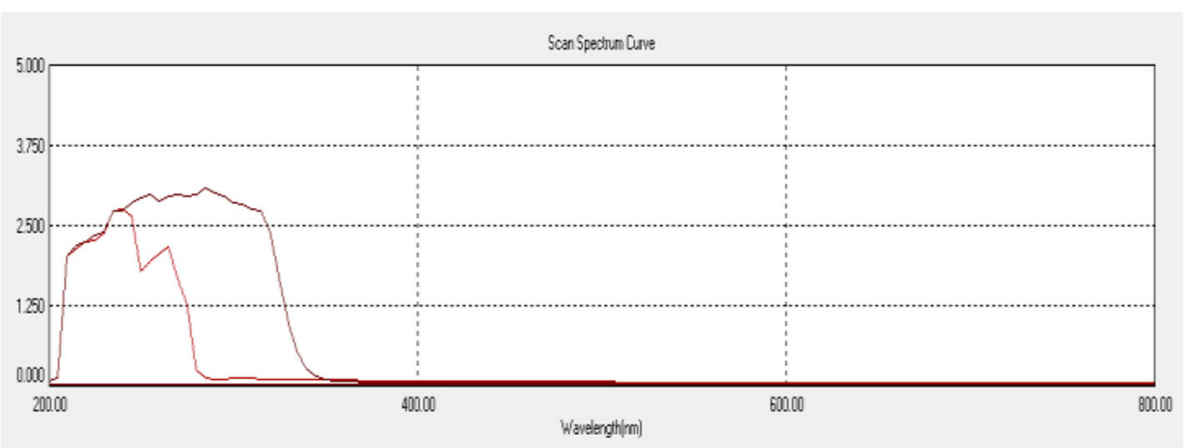

Fig. 4 UV-spectra of Tezacaftor and Ivacaftor

$5000 \mathrm{rpm}$ for $20 \mathrm{~min}$. The supernatant was collected and made up to $10 \mathrm{~mL}$ to get a final concentration $30 \mu \mathrm{g} / \mathrm{mL}$ of Tezacaftor and $45 \mu \mathrm{g} / \mathrm{mL}$ of Ivacaftor and was vortexed for $1 \mathrm{~min}$. These were filtered through 0.45 - $\mu$-pore size filters.

\section{Optimized chromatographic conditions}

Column: C18, Inspire ${ }^{\mathrm{tm}}(4.6 \times 250 \mathrm{~mm}, 5 \mu \mathrm{m})$

Mobile phase: Methanol: $0.05 \%$ formic acid (95:05)

Flow rate: $1.00 \mathrm{~mL} / \mathrm{min}$

Detector wavelength: $235 \mathrm{~nm}$

Column temperature: $30{ }^{\circ} \mathrm{C}$

Injection volume: $20 \mu \mathrm{l}$

The working standard solutions in mobile phase and in human plasma were used for optimization of method development and validation parameters as per $\mathrm{ICH}$ guidelines (Fig. 5a, b)

\section{Validation}

Linearity

Standard stock solutions containing $100 \mu \mathrm{g} / \mathrm{mL}$ of Tezacaftor and $150 \mu \mathrm{g} / \mathrm{mL}$ of Ivacaftor were prepared. Aliquots of these solutions were further diluted to produce five different concentrations, correspondingly, to $10-50 \mu \mathrm{g} / \mathrm{mL}$ of Tezacaftor and $15-75 \mu \mathrm{g} / \mathrm{mL}$ of Ivacaftor in both mobile phase and human plasma. Calibration curves for the different concentrations versus peak area were plotted for Ivacaftor and Tezacaftor, in both mobile phase and human plasma, and the obtained data were subjected to regression analysis (Figs. 6 and $7 \mathrm{a}, \mathrm{b})$ and the results were tabulated in Table 1.

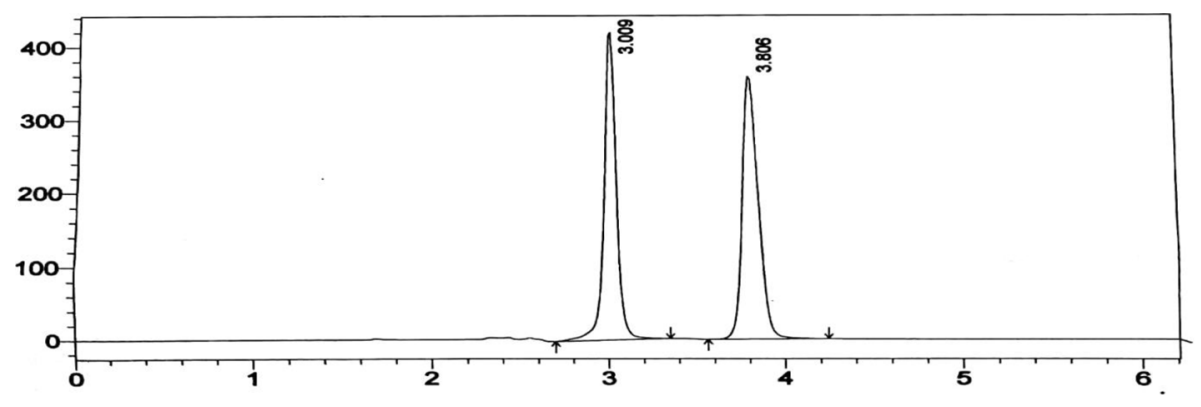

a. Optimized chromatogram of Tezacaftor and Ivacaftor in mobile phase

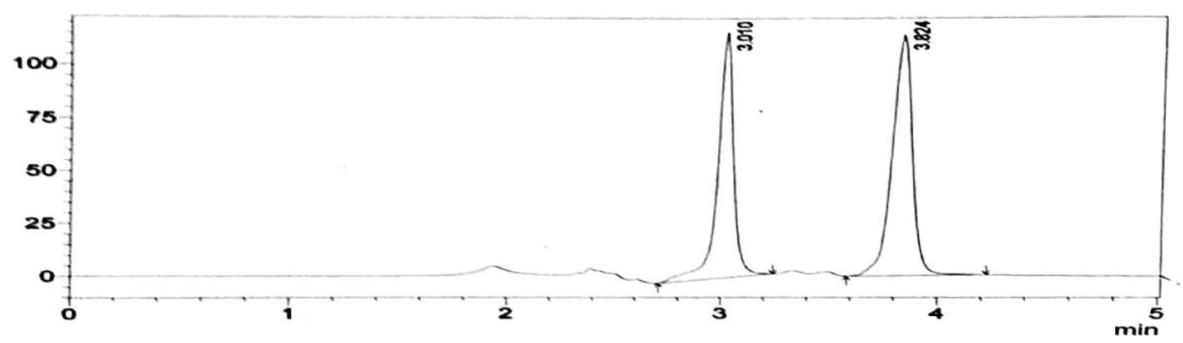

b. Optimized chromatogram of Tezacaftor and Ivacaftor in human plasma

Fig. 5 Optimised chromatograms of IVA and TEZ. a Optimized chromatogram. b Optimized chromatogram in mobile phase in human plasma 


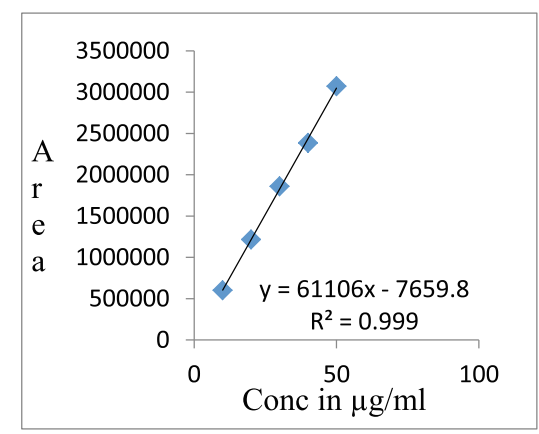

a) Linearity Curve of TEZ in Mobile phase

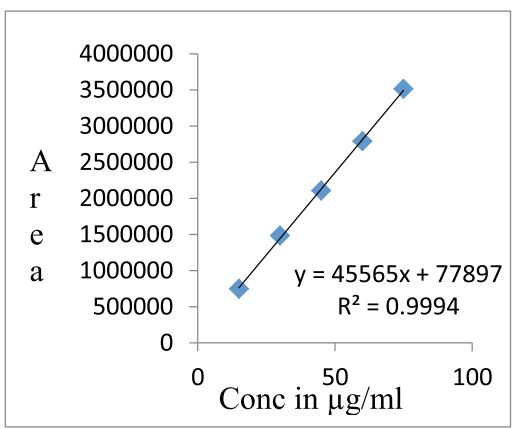

b) Linearity Curve of TEZ in Human plasma

Fig. $\mathbf{6}$ Linearity plot of Tezacaftor in mobile phase and human plasma respectively.a Linearity curve of TEZ in mobile phase. $\mathbf{b}$ Linearity curve of TEZ in human plasma

\section{Precision}

The intraday precision was evaluated by analyzing six solutions $(n=6)$, at the final concentration of analyses $30 \mu \mathrm{g} /$ $\mathrm{mL}$ of Tezacaftor and $45 \mu \mathrm{g} / \mathrm{mL}$ of Ivacaftor in both mobile phase and human plasma. Similarly, the interday precision was evaluated. Ivacaftor and Tezacaftor were determined and the \%R.S.D were calculated. Tables 2 and 3 results of the intermediate precision were tabulated in Tables 4 and 5

\section{Accuracy}

Ivacaftor and Tezacaftor reference standards were accurately weighed and added to a sample mixture at three different concentration levels $(15 \mu \mathrm{g} / \mathrm{mL}, 30 \mu \mathrm{g} / \mathrm{mL}$, and $45 \mu \mathrm{g} / \mathrm{mL}$ of Tezacaftor and $22.5 \mu \mathrm{g} / \mathrm{mL}, 45 \mu \mathrm{g} / \mathrm{mL}$, and $66.545 \mu \mathrm{g} / \mathrm{mL}$ of Ivacaftor). At each level, samples were prepared in triplicate and the recovery percentage was determined in both mobile phase and human plasma (Tables 6, 7, 8, and 9)

\section{Robustness}

Sample solution in both mobile phase and in human plasma were prepared and analyzed under the established conditions and by variation of the following analytical parameters flow rate of mobile phase $(0.8 \mathrm{~mL} /$ min, $1.2 \mathrm{~mL} / \mathrm{min})$, column temperature $\left(25{ }^{\circ} \mathrm{C}, 35^{\circ} \mathrm{C}\right)$ and detection wavelength $(225 \mathrm{~nm}, 245 \mathrm{~nm})$. Ivacaftor and Tezacaftor were determined for each condition and the obtained data were submitted for statistical analysis (Table 10).

\section{Detection and quantitation of limits}

Lowest concentration of sample solution in human plasma, $(10 \mu \mathrm{g} / \mathrm{mL}$ of Tezacaftor and $15 \mu \mathrm{g} / \mathrm{mL}$ of Ivacaftor) were injected in to HPLC and based on standard deviation of the response and the slope. The LOD and LOQ are calculated according to $\mathrm{ICH}$ guidelines (Table 11).

$$
\begin{aligned}
& \mathrm{LOD}=3.3 \times \mathrm{N} / \mathrm{S} \\
& \mathrm{LOQ}=10 \times \mathrm{N} / \mathrm{S}
\end{aligned}
$$

where $N$ is the SD of peak area of drug, $S$ is the slope of corresponding calibration curve.

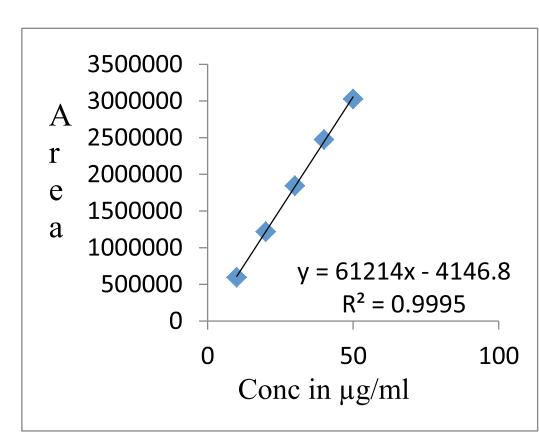

a) Linearity Curve of IVA in Mobile phase

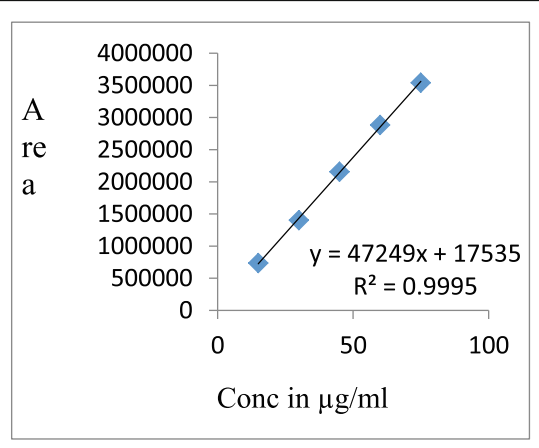

b) Linearity Curve of IVAin Human plasma

Fig. 7 Linearity plot of Ivacaftor in mobile phase and human plasma respectively.a Linearity curve of IVA in mobile phase. $\mathbf{b}$ Linearity curve of IVA in human plasma 
Table 1 Various parameters used in validation of Ivacaftor and Tezacaftor linearity studies

\begin{tabular}{|c|c|c|c|c|}
\hline \multirow[t]{2}{*}{ Parameters } & \multicolumn{2}{|l|}{ TEZ } & \multicolumn{2}{|l|}{ IVA } \\
\hline & Mobile phase & Human plasma & Mobile phase & Human plasma \\
\hline Wave length & $235 \mathrm{~nm}$ & $235 \mathrm{~nm}$ & $235 \mathrm{~nm}$ & $235 \mathrm{~nm}$ \\
\hline Concentration range & $10 \mu \mathrm{g} / \mathrm{mL}$ & $10 \mu \mathrm{g} / \mathrm{mL}$ & $15 \mu \mathrm{g} / \mathrm{mL}$ & $15 \mu \mathrm{g} / \mathrm{mL}$ \\
\hline Regression equation & $61106 x-7659.8$ & $45565 x+77897$ & $61214 x-4146.8$ & $47249 x+17535$ \\
\hline Regression coefficient & 0.999 & 0.9994 & 0.9995 & 0.995 \\
\hline
\end{tabular}

\section{Assay of fixed dose combination in placebo mixture}

Mixture was prepared by mixing drugs with placebo according to tablet composition of Symdeko. A portion of powder, equivalent to $100 \mathrm{mg}$ of Tezacaftor and $150 \mathrm{mg}$ of Ivacaftor was accurately weighed and transferred into $100 \mathrm{~mL}$ volumetric flask, followed by addition of $100 \mathrm{~mL}$ ACN. The solution was sonicated for $30 \mathrm{~min}$ and diluted with $\mathrm{ACN}$ to volume. Further dilutions were made to get final concentration equivalent to $30 \mu \mathrm{g} / \mathrm{mL}$ of Tezacaftor and $45 \mu \mathrm{g} / \mathrm{mL}$ of Ivacaftor and injected into HPLC after filtering through $0.45 \mu$ filter (Table 12).

\section{In silico docking studies}

\section{Molecular docking studies}

Molecular docking studies were carried out between Ivacaftor [1], Tezacaftor [2], their metabolites [15], and pathological proteins [16] which are involved in cystic fibrosis and they are CFTR Protein which were downloaded from PDB were docked with the abovementioned pathological proteins using PyRx [17]. The results were visualized using PyMol and Discovery studio visualizer (Discovery studio visualizer ver. 2.5) (Fig. 8a, b) (Tables 13 and 14).

\section{admetSAR predictions}

The pharmacokinetic properties such as Swiss ADME and PKCSM of the Ivacaftor and its metabolites Ivacaftor M1, M6 and Tezacaftor and metabolites Tezacaftor M1, M2 can be predicted [18]. In admetSAR, web-based query tools incorporating a molecular built-in interface enable the database to be queried by SMILES and structural similarity search. It provides the latest and most comprehensive manually curated data for diverse chemicals associated with known ADMET profiles (Tables 15, 16, 17, and 18).

\section{Toxicity prediction of potential metabolites Molinspiration}

This online tool helps the end user to analyze the molecular description and drug likeliness properties of compounds Molinspiration server works based on the Lipinski Rules of Five [19, 20]. The most "druglike" compound must possess the following properties: $\log \mathrm{P} \leq 5$, molecular weight $\leq 500 \mathrm{Da}$, number of hydrogen bond acceptors $\leq 10$, and number of hydrogen bond donors $\leq 5$. Compounds that failed to show these characters are least considered as a drug. Molinspiration server calculates the important molecular properties of compounds based on partition coefficient $(\log \mathrm{P})$, polar surface area, number of hydrogen bond donors and acceptors, and also prediction of bioactivity score for the drug targets such as G-protein-coupled receptor (GPCR) ligands, kinase, and protease inhibitors (EIs and PIs), ion channel modulators (ICMs), and nuclear receptor ligand (NRL). Topographical polar surface area (TPSA) was used to calculate the percentage of absorption using the following equation [21] (Tables 19 and 20).

$$
\text { Percentage of absorbance }=109-0.345 \times \text { TPSA }
$$

Table 2 Precision table of Tezacaftor in Mobile phase and Human plasma

\begin{tabular}{|c|c|c|c|c|c|c|c|}
\hline \multirow[t]{2}{*}{ S. no. } & \multirow{2}{*}{$\begin{array}{l}\text { Conc } \\
(\mu \mathrm{g} / \mathrm{mL})\end{array}$} & \multicolumn{3}{|l|}{ Mobile phase } & \multicolumn{3}{|l|}{ Human plasma } \\
\hline & & Conc found $(\mu \mathrm{g} / \mathrm{mL})$ & $\%$ & $\overline{\text { Avg } \%}$ & Conc found $(\mu \mathrm{g} / \mathrm{mL})$ & $\%$ & Avg \% \\
\hline 1 & 30 & 30.9 & 103 & 102.5 & 30.12 & 100.4 & 99.66 \\
\hline 2 & & 30.63 & 102.1 & & 29.96 & 99.86 & \\
\hline 3 & & 30.64 & 102.1 & & 29.99 & 99.96 & \\
\hline 4 & & 30.70 & 102.3 & & 29.84 & 99.46 & \\
\hline 5 & & 30.90 & 103 & & 29.68 & 98.93 & \\
\hline 6 & & 30.83 & 102.7 & & 29.82 & 99.4 & \\
\hline S.D & 0.05 & & & 0.098 & & & \\
\hline \% R.S.D & 0.16 & & & 0.32 & & & \\
\hline
\end{tabular}


Table 3 Precision table of Ivacaftor in mobile phase and human plasma

\begin{tabular}{|c|c|c|c|c|c|c|c|}
\hline \multirow[t]{2}{*}{ S. no. } & \multirow{2}{*}{$\begin{array}{l}\text { Conc } \\
(\mu \mathrm{g} / \mathrm{mL})\end{array}$} & \multicolumn{3}{|l|}{ Mobile phase } & \multicolumn{3}{|c|}{ Human plasma } \\
\hline & & $\begin{array}{l}\text { Conc found } \\
(\mu \mathrm{g} / \mathrm{mL})\end{array}$ & $\%$ & Avg \% & $\begin{array}{l}\text { Conc found } \\
(\mu \mathrm{g} / \mathrm{mL})\end{array}$ & $\%$ & Avg \% \\
\hline 1 & 45 & 44.99 & 99.9 & 100.06 & 45.95 & 102.1 & 100.02 \\
\hline 2 & & 44.78 & 99.5 & & 44.71 & 99.35 & \\
\hline 3 & & 44.99 & 99.9 & & 45.07 & 100.1 & \\
\hline 4 & & 44.88 & 99.7 & & 43.76 & 97.24 & \\
\hline 5 & & 45.29 & 100.6 & & 45.05 & 100.1 & \\
\hline 6 & & 45.39 & 100.8 & & 45.53 & 101.1 & \\
\hline S.D & & 0.096 & & & 0.484 & & \\
\hline \% R.S.D & & 0.21 & & & 1.07 & & \\
\hline
\end{tabular}

Table 4 Intermediate precision of Tezacaftor in mobile phase and human plasma

\begin{tabular}{|c|c|c|c|c|c|c|c|}
\hline \multirow[t]{2}{*}{ S. no. } & \multirow{2}{*}{$\begin{array}{l}\text { Conc } \\
(\mu \mathrm{g} / \mathrm{mL})\end{array}$} & \multicolumn{3}{|c|}{ Mobile phase } & \multicolumn{3}{|c|}{ Human plasma } \\
\hline & & $\begin{array}{l}\text { Conc found } \\
(\mu \mathrm{g} / \mathrm{mL})\end{array}$ & $\%$ & Avg \% & $\begin{array}{l}\text { Conc found } \\
(\mu \mathrm{g} / \mathrm{mL})\end{array}$ & $\%$ & Avg \% \\
\hline 1 & 30 & 30.7 & 102.3 & 101.5 & 30.69 & 102.3 & 101.8 \\
\hline 2 & & 30.52 & 101.7 & & 30.48 & 101.6 & \\
\hline 3 & & 30.22 & 100.7 & & 30.57 & 101.9 & \\
\hline 4 & & 30.32 & 101.0 & & 30.50 & 101.6 & \\
\hline 5 & & 30.49 & 101.6 & & 30.55 & 101.8 & \\
\hline 6 & & 30.52 & 101.7 & & 30.52 & 101.7 & \\
\hline S.D & & 0.021 & & & 0.03 & & \\
\hline \% R.S.D & & 0.06 & & & 0.09 & & \\
\hline
\end{tabular}

Table $\mathbf{5}$ Intermediate precision of Ivacaftor in mobile phase and human plasma

\begin{tabular}{|c|c|c|c|c|c|c|c|}
\hline \multirow[t]{2}{*}{ S. no. } & \multirow{2}{*}{$\begin{array}{l}\text { Conc } \\
(\mu \mathrm{g} / \mathrm{mL})\end{array}$} & \multicolumn{3}{|c|}{ Mobile phase } & \multicolumn{3}{|c|}{ Human plasma } \\
\hline & & $\begin{array}{l}\text { Conc found } \\
(\mu \mathrm{g} / \mathrm{mL})\end{array}$ & $\%$ & Avg \% & $\begin{array}{l}\text { Conc found } \\
(\mu \mathrm{g} / \mathrm{mL})\end{array}$ & $\%$ & Avg $\%$ \\
\hline 1 & 45 & 46.53 & 103.4 & 99.15 & 45.94 & 102.0 & 101.7 \\
\hline 2 & & 44.6 & 99.1 & & 46.01 & 102.2 & \\
\hline 3 & & 44.26 & 98.3 & & 45.68 & 101.5 & \\
\hline 4 & & 44.54 & 98.9 & & 45.71 & 101.5 & \\
\hline 5 & & 43.84 & 97.4 & & 45.72 & 101.6 & \\
\hline 6 & & 44.04 & 97.8 & & 45.68 & 101.5 & \\
\hline S.D & & 0.396 & & & & & \\
\hline \% R.S.D & & 0.8 & & & & & \\
\hline
\end{tabular}


Table $6 \%$ Recovery of Tezacaftor in mobile phase

\begin{tabular}{|c|c|c|c|c|c|c|}
\hline S. no. & $\%$ Level & Conc added $(\mu \mathrm{g} / \mathrm{mL})$ & \% Recovery & Average $\%$ & S.D & $\%$ RSD \\
\hline 1 & $50 \%$ & 15 & 97.3 & 99.3 & 0.27 & 0.61 \\
\hline 2 & & & 103 & & & \\
\hline 3 & & & 97.6 & & & \\
\hline 4 & $100 \%$ & 30 & 101.73 & 102.86 & 0.17 & 0.27 \\
\hline 5 & & & 103.46 & & & \\
\hline 6 & & & 103.4 & & & \\
\hline 7 & $150 \%$ & 45 & 98 & 97.75 & 0.07 & 0.09 \\
\hline 8 & & & 97.82 & & & \\
\hline 9 & & & 97.44 & & & \\
\hline
\end{tabular}

Table 7 \%ecovery of Tezacaftor in human plasma

\begin{tabular}{|c|c|c|c|c|c|c|}
\hline S. no. & $\%$ Level & Conc added $(\mu \mathrm{g} / \mathrm{mL})$ & \% Recovery & Average $\%$ & S.D & $\%$ RSD \\
\hline 1 & $50 \%$ & 15 & 98.2 & 98.23 & 0.023 & 0.05 \\
\hline 2 & & & 98 & & & \\
\hline 3 & & & 98.5 & & & \\
\hline 4 & $100 \%$ & 30 & 97.93 & 98.16 & 0.03 & 0.05 \\
\hline 5 & & & 98.36 & & & \\
\hline 6 & & & 98.2 & & & \\
\hline 7 & $150 \%$ & 45 & 96.9 & 97.6 & 0.35 & 0.47 \\
\hline 8 & & & 96.8 & & & \\
\hline 9 & & & 99.17 & & & \\
\hline
\end{tabular}

Table 8 \%Recovery of Ivacaftor in mobile phase

\begin{tabular}{|c|c|c|c|c|c|c|}
\hline S.No & $\%$ Level & Conc added $(\mu \mathrm{g} / \mathrm{mL})$ & \% Recovery & Average $\%$ & S.D & $\%$ RSD \\
\hline 1 & $50 \%$ & 22.5 & 101.77 & 101.75 & 0.21 & 0.3 \\
\hline 2 & & & 100.08 & & & \\
\hline 3 & & & 103.42 & & & \\
\hline 4 & $100 \%$ & 45 & 103.8 & 103.93 & 0.33 & 0.36 \\
\hline 5 & & & 102.7 & & & \\
\hline 6 & & & 105.3 & & & \\
\hline 7 & $150 \%$ & 67.5 & 101.3 & 100.32 & 0.34 & 0.3 \\
\hline 8 & & & 100.1 & & & \\
\hline 9 & & & 99.58 & & & \\
\hline
\end{tabular}


Table $9 \%$ Recovery of Ivacaftor in human plasma

\begin{tabular}{|c|c|c|c|c|c|c|}
\hline S. no. & $\%$ Level & Conc added $(\mu \mathrm{g} / \mathrm{mL})$ & \% Recovery & Average $\%$ & S.D & $\%$ RSD \\
\hline 1 & $50 \%$ & 22.5 & 97.46 & 97.44 & 0.001 & 0.002 \\
\hline 2 & & & 97.4 & & & \\
\hline 3 & & & 97.42 & & & \\
\hline 4 & $100 \%$ & 45 & 103.35 & 103.5 & 0.10 & 0.11 \\
\hline 5 & & & 104.06 & & & \\
\hline 6 & & & 103.37 & & & \\
\hline 7 & $150 \%$ & 67.5 & 98.59 & 98.04 & 0.28 & 0.25 \\
\hline 8 & & & 98.31 & & & \\
\hline 9 & & & 97.22 & & & \\
\hline
\end{tabular}

Table 10 Robustness study of Tezacaftor and Ivacaftor in Mobile phase and Human plasma

\begin{tabular}{|c|c|c|c|c|c|c|c|}
\hline \multirow{2}{*}{$\begin{array}{l}\text { S. } \\
\text { no. }\end{array}$} & \multirow[t]{2}{*}{ Parameters } & \multirow[t]{2}{*}{ Normal } & \multirow[t]{2}{*}{ Variation } & \multicolumn{2}{|c|}{$\%$ RSD in mobile phase } & \multicolumn{2}{|c|}{ \%RSD in human plasma } \\
\hline & & & & Tezacaftor & Ivacaftor & Tezacaftor & Ivacaftor \\
\hline \multirow[t]{2}{*}{1} & Wavelength & $235 \mathrm{~nm}$ & $225 \mathrm{~nm}$ & 0.77 & 0.8 & 0.38 & 0.54 \\
\hline & & & $245 \mathrm{~nm}$ & 0.2 & 0.03 & 0.36 & 0.106 \\
\hline \multirow[t]{2}{*}{2} & Temperature & $30^{\circ} \mathrm{C}$ & $25^{\circ} \mathrm{C}$ & 1.035 & 1.7 & 0.24 & 0.44 \\
\hline & & & $35^{\circ} \mathrm{C}$ & 0.76 & 0.58 & 0.15 & 0.38 \\
\hline \multirow[t]{2}{*}{3} & Flow Rate & $1 \mathrm{~mL} / \mathrm{min}$ & $0.8 \mathrm{~mL} / \mathrm{min}$ & 0.64 & 0.13 & 0.298 & 0.22 \\
\hline & & & $1.2 \mathrm{~mL} / \mathrm{min}$ & 0.125 & 0.06 & 0.02 & 0.06 \\
\hline
\end{tabular}

Table 11 LOD and LOQ study of Tezacaftor and Ivacaftor in mobile phase and human plasma

\begin{tabular}{lllll}
\hline $\begin{array}{llll}\text { Name of } \\
\text { the drug }\end{array}$ & Mobile phase & Human plasma \\
\cline { 2 - 5 } & $\mathrm{LOD}(\mu \mathrm{g} / \mathrm{mL})$ & $\mathrm{LOQ}(\mu \mathrm{g} / \mathbf{m L})$ & $\mathrm{LOD}(\mu \mathrm{g} / \mathbf{m L})$ & $\mathrm{LOQ}(\mu \mathrm{g} / \mathbf{m L})$ \\
\hline Tezacaftor & 0.011 & 0.03 & 0.09 & 0.275 \\
Ivacaftor & 0.115 & 0.349 & 0.31 & 0.96 \\
\hline
\end{tabular}

Table 12 Assay studies of Tezacaftor and Ivacaftor in mobile phase and human plasma

\begin{tabular}{|c|c|c|c|c|c|c|c|}
\hline \multirow{2}{*}{$\begin{array}{l}\text { Name of } \\
\text { the drug }\end{array}$} & \multirow{2}{*}{$\begin{array}{l}\text { Label } \\
\text { claim } \\
(\mathrm{mg})\end{array}$} & \multicolumn{3}{|l|}{ Mobile phase } & \multicolumn{3}{|l|}{ Human plasma } \\
\hline & & Amount found (mg) & Assay \% & $\%$ RSD & Amount found (mg) & Assay \% & $\%$ RSD \\
\hline Tezacaftor & 30 & 30.275 & 101.01 & 1.172 & 30.41 & 101.35 & 0.625 \\
\hline Ivacaftor & 45 & 45.3 & 100.71 & 1.23 & 44.925 & 99.8 & 1.57 \\
\hline
\end{tabular}


a) Binding of Ivacaftor to CFTR protein

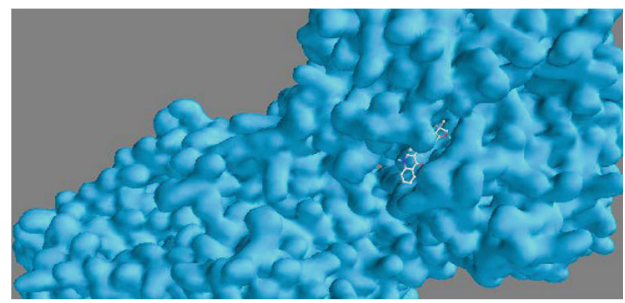

b) Binding of Ivacaftor M1 to CFTR protein

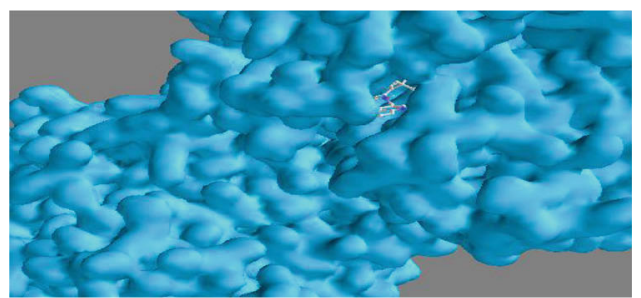

c) Binding of Ivacaftor M6 to CFTR protein

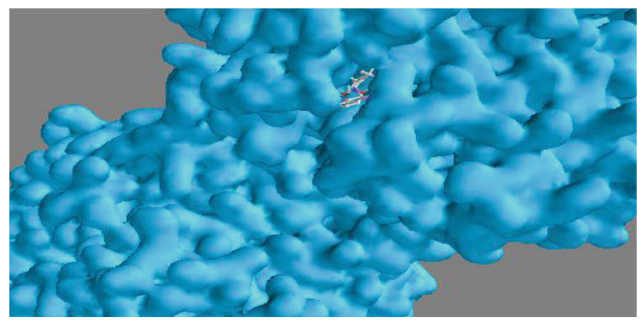

d) Binding of Tezacaftor to CFTR protein

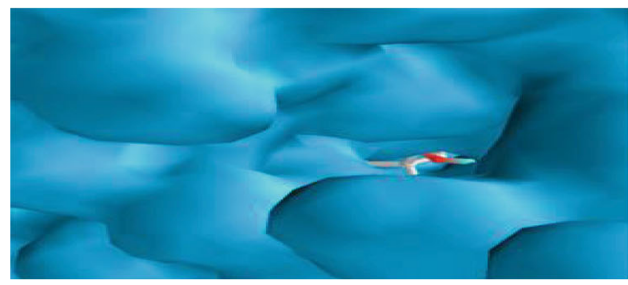

e) Binding of Tezacaftor M1 to CFTR protein

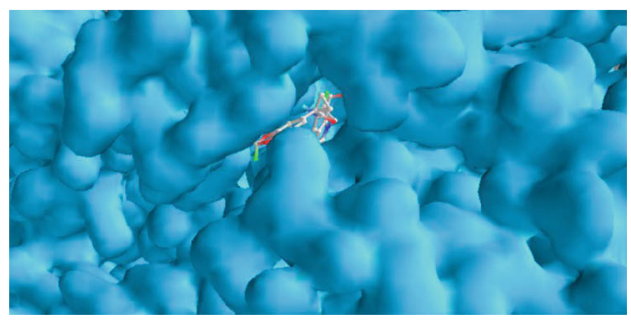

f) Binding of Tezacaftor M2 to CFTR protein

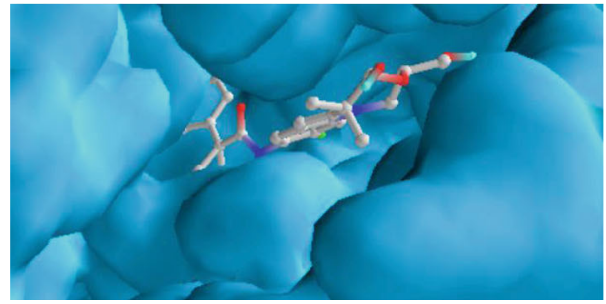

Fig. 8 In silico docking studies by PyRx tool. a Binding of Ivacaftor to CFTR protein. $\mathbf{b}$ Binding of Ivacaftor M1 to CFTR protein. c Binding of Ivacaftor M6 to CFTR protein. d Binding of Tezacaftor to CFTR protein. e Binding of Tezacaftor M1 to CFTR protein. $\mathbf{f}$ Binding of Tezacaftor M2 to CFTR protein

\section{Biological activity spectrum (BAS)}

Biological activity spectrum (BAS) of a compound represents an intrinsic property of the pharmacological effects, physiological and biochemical mechanisms of action, and specific toxicity (mutagenicity, carcinogenicity, teratogenicity, and embryotoxicity), which is largely dependent on the interaction with the biological system $[22,23]$. The values vary from 0.000 to 1.000 . Only those activity types for which $\mathrm{Pa}$ (probability to be active) $>\mathrm{Pi}$ (probability to be inactive) were considered possible [24, 25](Table 21).

\section{Results}

\section{Method development}

Chromatographic parameters were optimized to develop a HPLC method for the simultaneous estimation of Ivacaftor and Tezacaftor in both mobile phase and in human plasma, with short analysis time $(<5 \mathrm{~min})$ and acceptable resolution $\left(R_{\mathrm{s}}>2\right)$ various composition of mobile phase like ACN:1\% IPA (95:05), ACN: 1\%I.P.A(80:20), ACN; $0.5 \%$ formic acid (70:30), ACN: $0.5 \%$ formic acid (90:10), ACN: 0.1\% formic acid (85:15), ACN:0.1\% formic acid (90:10), were tried at flow rate of $1 \mathrm{~mL} / \mathrm{min}$. Methanol and $0.05 \%$ formic acid in ratio of 95.5 showed symmetrical peaks with good resolution in both mobile phase and human plasma. The optimum wavelength for detection was $235 \mathrm{~nm}$ and the RT was found to be $3.0 \mathrm{~min}$ and $3.8 \mathrm{~min}$ for Tezacaftor and Ivacaftor, respectively. Linearity curve was obtained in the concentration range of $10-50 \mu \mathrm{g} / \mathrm{mL}$ of Tezacaftor and $15-75 \mu \mathrm{g} / \mathrm{mL}$ of Ivacaftor. LOD and LOQ were determined from the slope and SD of Y-intercept of regression line of the calibration curve. The precision of the method and instrument precision were evaluated, and the \%R.S.D values were less than $2 \%$ human plasma. The accuracy of the method was determined by recovery studies. The recovery studies were close to $100 \%$ which complies with ICH guidelines and FDA approval. Developed method was found to robust while changing the flow rate wavelength detection and temperature. The proposed method is superior when compared to the reported method with less RT and good separation and can be applied for biological fluids.

\section{In silico study}

The Lipinski "rule of five" is commonly used as an index during drug design and development to predict the oral 
Table 13 Molinspiration calculation of Ivacaftor and its metabolites

\begin{tabular}{|c|c|c|c|c|c|c|}
\hline Name & miLogP & TPSA & Natoms & MW & nrotb & Volume \\
\hline Ivacaftor & 4.78 & 82.19 & 29 & 392.5 & 4 & 375.19 \\
\hline Ivacaftor M1 & 3.6 & 102.42 & 30 & 408.5 & 5 & 383.45 \\
\hline Ivacaftor M6 & 3.47 & 119.49 & 31 & 422.48 & 5 & 385.63 \\
\hline
\end{tabular}

Table 14 Molinspiration calculation of Tezacaftor and its metabolites

\begin{tabular}{lllllll}
\hline Name & miLogP & TPSA & Natoms & MW & Nrotb & Volume \\
\hline Tezacaftor & 2.8 & 111.49 & 36 & 510.51 & 435.38 \\
Tezacaftor M1 & 3.97 & 102.19 & 37 & 518.49 & 4 & 424.95 \\
Tezacaftor M2 & 3.39 & 130.25 & 38 & 534.49 & 8 & 436.98 \\
\hline
\end{tabular}

Table 15 Bioactivities of Ivacaftor and its metabolites based on the drug likeness score were calculated using Molinspiration tool

\begin{tabular}{lllllll}
\hline Name & GPCR ligand & ICM & KI & NRL & PI & El \\
\hline Ivacaftor & -0.07 & -0.14 & 0.15 & 0 & -0.28 & 0.07 \\
Ivacaftor M1 & 0 & 0 & 0.26 & 0.03 & -0.2 & 0.1 \\
Ivacaftor M6 & 0.04 & -0.12 & 0.18 & 0.18 & -0.16 & 0.17 \\
\hline
\end{tabular}

Table 16 Bioactivities of Tezacaftor and its metabolites based on the drug likeness score were calculated using Molinspiration tool

\begin{tabular}{|c|c|c|c|c|c|c|}
\hline Name & GPCR ligand & ICM & $\mathrm{KI}$ & NRL & $\mathrm{PI}$ & EI \\
\hline Tezacaftor & 0.3 & -0.06 & -0.27 & 0.08 & 0.34 & 0.17 \\
\hline Tezacaftor M1 & 0.21 & -0.28 & 0.09 & 0.03 & 0.21 & 0.20 \\
\hline Tezacaftor M2 & 0.25 & -0.29 & 0.08 & 0.06 & 0.17 & 0.10 \\
\hline
\end{tabular}


Table 17 Predicted molecular pharmacokinetic properties of Ivacaftor and its metabolites using Swiss ADME database

\begin{tabular}{lllllllll}
\hline Name & Gl absorption & BBB permeant & P-gp substrate & $\begin{array}{l}\text { CYP1A2 } \\
\text { Inhibitor }\end{array}$ & $\begin{array}{l}\text { CYP2C19 } \\
\text { Inhibitor }\end{array}$ & $\begin{array}{l}\text { CYP2C9 } \\
\text { Inhibitor }\end{array}$ & $\begin{array}{l}\text { CYP2D6 } \\
\text { Inhibitor }\end{array}$ & $\begin{array}{l}\text { CYP3A4 } \\
\text { Inhibitor }\end{array}$ \\
\hline Ivacaftor & High & No & No & No & Yes & No & No & Yes \\
Ivacaftor M1 & High & No & No & Yes & No & No & Yes & No \\
Ivacaftor M6 & High & No & No & Yes & No & No & No & No \\
\hline
\end{tabular}

Table 18 Predicted molecular pharmacokinetic properties of Tezacaftor and their metabolites using Swiss ADME database

\begin{tabular}{lllllllll}
\hline Name & Gl absorption & BBB permeant & P-gp substrate & $\begin{array}{l}\text { CYP1A2 } \\
\text { Inhibitor }\end{array}$ & $\begin{array}{l}\text { CYP2C19 } \\
\text { Inhibitor }\end{array}$ & $\begin{array}{l}\text { CYP2C9 } \\
\text { Inhibitor }\end{array}$ & $\begin{array}{l}\text { CYP2D6 } \\
\text { Inhibitor }\end{array}$ & $\begin{array}{l}\text { CYP3A4 } \\
\text { Inhibitor }\end{array}$ \\
\hline Tezacaftor & High & No & Yes & No & No & No & No & No \\
Tezacaftor M1 & High & No & Yes & No & No & Yes & Yes & Yes \\
Tezacaftor M2 & Low & No & Yes & No & No & Yes & Yes & Yes \\
\hline
\end{tabular}

Table 19 Predicted molecular toxicity of Ivacaftor and its metabolites using data warrior

\begin{tabular}{llllllll}
\hline Name & Mutagenic & Tumorigenic & Reproductive & Irritant & ORAT & ORCT & Hepatotoxicity \\
\hline Ivacaftor & None & None & None & None & $2.073 \mathrm{~mol} / \mathrm{kg}$ & $2.022 \mathrm{log} \mathrm{mg} / \mathrm{kg}$ & Yes \\
Ivacaftor M1 & None & None & None & None & $2.149 \mathrm{~mol} / \mathrm{kg}$ & $2.298 \mathrm{log} \mathrm{mg} / \mathrm{kg}$ & Yes \\
Ivacaftor M6 & None & None & None & None & $2.428 \mathrm{~mol} / \mathrm{kg}$ & $2.826 \mathrm{log} \mathrm{mg} / \mathrm{kg}$ & Yes \\
\hline
\end{tabular}

Table 20 Predicted molecular toxicity of Tezacaftor and its metabolites using data warrior

\begin{tabular}{|c|c|c|c|c|c|c|c|}
\hline Name & Mutagenic & Tumorigenic & Reproductive & Irritant & ORAT & ORCT & Hepatotoxicity \\
\hline Tezacaftor & None & None & None & High & $3.732 \mathrm{~mol} / \mathrm{kg}$ & $2.997 \mathrm{log} \mathrm{mg} / \mathrm{kg}$ & Yes \\
\hline Tezacaftor M1 & None & None & None & High & $2.963 \mathrm{~mol} / \mathrm{kg}$ & $1.814 \mathrm{log} \mathrm{mg} / \mathrm{kg}$ & Yes \\
\hline Tezacaftor M2 & None & None & None & High & $2.966 \mathrm{~mol} / \mathrm{kg}$ & $3.963 \mathrm{log} \mathrm{mg} / \mathrm{kg}$ & Yes \\
\hline
\end{tabular}


Table 21 Biological spectrum of Ivaacaftor, Tezacaftor, and its metabolites

\begin{tabular}{lllllll}
\hline Probability & Ivacaftor & Ivacaftor $\mathbf{M}$ 1 & Ivacaftor M6 & Tezacaftor & Tezacaftor M1 & Tezacaftor M2 \\
\hline $\mathrm{Pa}$ & 0.609 & 0.534 & 0.589 & 0.265 & 0.463 & 0.600 \\
$\mathrm{Pi}$ & 0.003 & 0.003 & 0.003 & 0.075 & 0.005 & 0.002
\end{tabular}

bioavailability of the lead drug molecules. Based on Lipinski's "rule of five," drug compound should satisfy the following criteria: (1) the molecular weight of the candidate drug should be $<500,(2) \log P<5$, and (3) TPSA < 140, (4) natom (20-70), (5) nrob $(<10)$.

\section{Discussions}

A novel rapid method was developed for the simultaneous determination of Ivacaftor and Tezacaftor in human plasma was found to be linear, precise, accurate, and economical. The analytical conditions were optimized for short analysis time using formic acid as eluent during analysis in biological fluids. The acid is used to improve the chromatographic peak in presence of matrix, and it supplies proton to Tezacaftor which is having pKa value of 11.54. Formic acid has less molecular weight, due to its ion-pair nature, and volatility good resolution is obtained [24]. The \%R.S.D for all parameters was found to be within limits for both mobile phase and human plasma. The results obtained are in good agreement and can be used for the routine analysis of Ivacaftor and Tezacaftor in combined dosage form in laboratories and for quality control purpose. This method can be applied for the assay, determination of drugs in plasma at different time intervals, can be applied for bioavailability studies, and can analyze multiple samples in 1 day. This method is said to be on par with L.C/M.S method. Molecular docking studies results of Ivacaftor and its metabolites revealed that there is no significant change in the binding energies $(-8.3,-8.6,-8.7 \mathrm{Kcal}$, respectively). Molecular docking studies results of Tezacaftor and its metabolites revealed that there is a significant change in the binding energy $(-7.8,-10$, $10.8 \mathrm{Kcal}$, respectively). Hence, the biological activities and toxicity studies of drugs and their metabolites using Molinspiration, Swiss ADME database, and data warrior softwares were observed and that Ivacaftor has more biological activity and no prominent toxicity and Tezacaftor M2 has more biological activity.

\section{Conclusion}

From the results obtained, most parameters of Ivacaftor, Tezacaftor, and its metabolites were within the Lipinski Rule of Five. Hence, Ivacaftor and metabolites and Tezacaftor and its Metabolites do not violate the Lipinski rule and could be expected to be orally active as they followed Lipinski Rule of
Five. If a molecule is predicted to have a bioactivity score of $>0.00$, it is likely to demonstrate considerable biological activities, whereas the values $-0.50-0.00$ indicate moderately active molecules, and less than 0.50 is presumed to be inactive.

From the results, it is obvious that the physiological actions of Ivacaftor M1 could be due to the strong interactions with GPCR ligands, NRLs, ICM, KI, EI, and inhibition of proteases and other enzymes. These bioactivity scores obtained suggested that Ivacaftor M1 compounds could interact with all the drug targets. All the compounds showed good bioactivity based on the scores obtained for all drug targets.

The physiological actions of Tezacaftor could be due to the strong interactions with GPCR ligands, NRLs, ICM, PI, EI, and inhibition of proteases and other enzymes. These bioactivity scores obtained suggested that Tezacaftor M2 compounds could interact with all the drug targets. All the compounds showed good bioactivity based on the scores obtained for all drug targets.

ADMET properties of Ivacaftor and its metabolites Ivacaftor M1, M6 and Tezacaftor and its metabolites Tezacaftor M1, M2 were calculated using Swiss ADME and Pkcsm. Ivacaftor M1 inhibit the enzyme CYP1A2 and CYP2D6 and no inhibition of CYP2C19, CYP2C9, and CYP3A4 compared with Ivacaftor and no change in toxicity profile between Ivacaftor and its metabolites

Tezacaftor M1, M2 inhibit the enzyme CYP2C9, CYP2D6, and CYP3A4, and no inhibition of CYP1A2 and CYP2C19, compared with Tezacaftor which no inhibition in all cases and no change in toxicity profile between Tezacaftor and its metabolites. Ivacaftor use to treat cystic fibrosis. The obtained results also exhibit $\mathrm{Pa}>\mathrm{Pi}$ values for all the metabolites. Hence, BAS tools predict the biological activities of Ivacaftor and its metabolites treat cystic fibrosis. Tezacaftor use to treat cystic fibrosis. The obtained results also exhibit $\mathrm{Pa}>\mathrm{Pi}$ values for metabolites. Hence, BAS tools predict the biological activities of Tezcaftor and its metabolites in the treatment of cystic fibrosis.

\section{Abbreviations}

RP-HPLC: Reverse phase high-pressure liquid chromatography; TEZ: Tezacaftor; IVA: Ivacaftor; IVA M1: Ivacaftor metabolite 1; IVA M6: Ivacaftor metabolite 6; TEZ M1: Tezacaftor metabolite 1; TEZ M2: Tezacaftor metabolite 2; LOD: Limit of detection; LOQ: Limit of quantification; CFTR: Cystic fibrosis transmembrane conductance regulator; I.C.H.: International Conference on Harmonization; ACN: Acetonitrile; PDA: Photodiode array; PDB: Protein Data Bank; ADMET: Absorption distribution metabolism excretion and toxicity; pKCSM: Predicting small 
molecule pharmacokinetic and toxicity; GPCR: G-protein coupled receptor; ICM: Ion channel modulators; NRL: Nuclear receptor ligand;

TPSA: Topographical polar surface area; IPA: Isopropyl alcohol; SD: Standard deviation; RSD: Relative standard deviation; RT: Retention time

\section{Acknowledgements}

I would like to express our sincere thanks to the management and Principa Dr. P. Raviprakash of Creative Educational Society's College of Pharmacy for the facilities, requirements, and constant support during the research work.

\section{Nature recommended repositories}

Not applicable.

\section{Authors' contributions}

MD: plan and design of work. SI: experimental procedure for RP-HPLC method development and validation. PP: in silico docking studies and results of docking evaluation. M.R.D: experimental procedure for RP-HPLC method development and validation. S.K: in silico docking. All the authors have read and approved the final manuscript.

\section{Funding}

No funding was received.

\section{Availability of data and materials}

All data and materials are available upon request.

\section{Declarations}

Ethics approval and consent to participate

Not applicable.

\section{Consent for publication}

Not applicable.

\section{Competing interests}

The authors declare that they have no competing interests.

\section{Author details}

'Department of Pharmaceutical Analysis and Quality Assurance, CES College of Pharmacy, Chinnatekur, NH-7, Kurnool, Andhra Pradesh 518218, India. ${ }^{2}$ Department of Pharmacology, Santhiram College of Pharmacy, Kurnool, Andhra Pradesh 518501, India.

Received: 4 March 2020 Accepted: 26 April 2021

Published online: 07 June 2021

\section{References}

1. Compound summary of Ivacaftor https://pubchem.ncbi.nlm.nih.gov/ compound/16220172.

2. Rahan JW, Elizabethmatthes G, Ythomas D (2017) Corrector combination therapies for F508del-CFTR. Curr Opin Pharmacol 342017:105-111

3. Compound summary of Tezacaftor https://pubchem.ncbi.nlm.nih.gov/ compound/46199646

4. Kiranjyothi R, Balakrishnan M, Chandrasekhar KB (2018) Method development and validation for the stability indicating simultaneous estimation of Tezacaftorand Ivacaftor in bulk and its dosage forms. Int J Pharmaceut Res 10(4):198-208. 4

5. Sravanthi B, Divya M (2016) Analytical method development and validation of ivacaftor and lumacaftor by RP-HPLC method. Indo Am J Pharmaceut Sci 3(8):900-904

6. Gorantla N, Dodlapati J, Jadi S (2019) A new validated RP-HPLC method for simultaneous estimation of Lumacaftor and Ivacaftor in pharmaceutical dosage form. Int J Pharmaceut Sci Rev Res 56(1):30-37

7. Naresh SD, Sowjanya P, Kumar GV (2016) Analytical method development and validation for the simultaneous estimation of Ivacaftor and Lumacaftor in its bulk and pharmaceutical dosage forms. Int Natl J Med Pharmaceut Res 4(6):331-335

8. Suresh Babu M, Spandhana N, Babyrani P, Jagadheesh P, Akhil P (2017) Analytical method development and validation for the estimation of Lumacaftor and Ivacaftor using RP-HPLC. J Pharma Creations 4(1):55-78
9. Chhabda PJ, Balaji M, Srinivasarao V (2018) Development and validation of a new and stability indicating RP-HPLC method for the determination of Ivacaftor in presence of degradant products. Int J Pharm Pharm Sci 5(4):607-613

10. Rameeja P, Haribaskar V, Mounika PS, Ramesh D, Prathap B (2018) Stability indicating RP-HPLC method for simultaneous estimation of Lumacaftor and Ivacaftor in bulk and pharmaceutical dosage forms. Pharma Res Libr 6(10):273-278

11. Schneider EK, Felisareyes-Ortega, Wilson JW, Tomkotsimbos D, Jianli T (2016) Development of HPLC and LC-MS/MS methods for the analysis of Ivacaftor, its major metabolites and lumacaftor in plasma and sputum of cystic fibrosis patients treated with ORKAMBI or KALYDECO. J Chromatogr B 1038:57-62

12. Sonawane MD, Gade ST, Narwate BM (2018) Application and UV spectrophotometric method development and validation for simultaneous estimation of Tezacaftor and Ivacaftor in pharmaceutical dosage form. World J Pharmaceut Res 7(14):213-219

13. Gautam CVS, Sai Charan K, Swathi B, Mounika M (2019) Method development and validation of Ivacaftor in Bulk \& Pharmaceutical Dosage Form by UV-visible spectrophotometry. Indo Am J Pharmaceut Sci 6(4):7476-7481

14. Mohan Goud V, Sharma JVC, Sravanthi M (2019) Stability indicating ultra performance liquid chromatography method development and validation for simultaneous estimation of Ivacaftor and Tezacaftor in bulk and pharmaceutical dosage form. Int J Sci Res Rev 8(5):128-133

15. Núñez O, Lucci $P$ (2014) Applications and uses of formic acid in liquid chromatography-mass spectrometry analysis. Adv Chem Res 20:71-86

16. Compound summary of Ivacaftor and Tezacaftor https://pubchem.Ncbi.nIm. nih.gov/compound/72722243

17. Biological Macromolecular structures Enabling breakthroughs in research and Education www.rcsb.org/pdb

18. Trott O, Olson AJ (2010) AutoDock Vina: improving the speed and accuracy of docking with a new scoring function, efficient optimization, and multithreading. J Comput Chem 31(2):455-461

19. Pires DE, Blundell TL, Ascher DB (2015) pkCSM: predicting small-molecule pharmacokinetic and toxicity properties using graph-based signatures. J Med Chem 58(9):4066-4072. https://doi.org/10.1021/acs.jmedchem.5b00104

20. Prediction of probable activity spectra of substances. http://www.molinspira tion.com.www.pharmaexpert.ru/passonline/index.php.

21. Carlsson L, Spjuth O, Adams S, Glen RC, Boyer S (2010) Use of historic metabolic biotransformation data as a means of anticipating metabolic sites using metaPrint2D and bioclipse. BMC Bioinformatics 11(1):362. https://doi. org/10.1186/1471-2105-11-362

22. Lipinski CA, Lombardo F, Dominy BW, Feeney PJ (2001) Experimental and computational approaches to estimate solubility and permeability in drug discovery and development settings. Adv Drug Deliv Rev 46(1-3):3-26. https://doi.org/10.1016/S0169-409X(00)00129-0

23. Cheng F, Li W, Zhou Y, Shen J, Wu Z, Liu G (2012) AdmetSAR: a comprehensive source and free tool for assessment of chemical ADMET properties. J Chem Inf Model 52(11):3099-3105. https://doi. org/10.1021/ci300367a

24. Daina A, Michielin O, Zoete V (2017) Swiss ADME: a free web tool to evaluate pharmacokinetics, drug-likeness and medicinal chemistry friendliness of small molecules. Sci Rep 3(7):42717

25. Insilico screening approaches help to reduce risks a novel approach to the prediction of pharmacokinetic properties http://biosig.unimelb.edu.au/pkcsm/

\section{Publisher's Note}

Springer Nature remains neutral with regard to jurisdictional claims in published maps and institutional affiliations. 